\title{
Molecular Docking Analysis of Anti-malarial Compounds as Plasmepsin IV Inhibitor from Targeted Indonesian Medicinal Plants
}

\author{
Arthur Hariyanto Prakoso, Muhammad Habiburrohman, Wilda Nur Rohmatillah, \\ Bawon Triatmoko, Ari Satia Nugraha*
}

Drug Utilization and Discovery Research Group, Faculty of Pharmacy, University of Jember, Jember, Indonesia 68121

${ }^{*}$ Corresponding author:
E-mail: arisatia@unej.ac.id

\begin{abstract}
Malaria is one of the major causes of death in tropical and sub-tropical countries, caused by the infection of the protozoan parasite (Plasmodium falciparum, Plasmodium vivax, Plasmodium malariae, and Plasmodium knowlesi). As the prevalence of parasite drug-resistant strains increasing, alternative medicine to eliminate malaria is needed. In this study, a molecular docking protocol was employed to predict and select natural compounds from Indonesian medicinal plants as an antimalarial drug candidate. The docking protocol was validated by the RMSD value of crystal versus docking calculation. From 43 species of plants, 238 total compounds were collected and docked into Plasmepsin IV (PMIV) enzyme which plays a role in the nutrition uptake of Plasmodium in human blood circulation. This enzyme was collected from a protein database with codename 5I70. The protocol was produced an acceptable RMSD value of $1.435 \AA$ The docking experiment resulted in AM202 (Cassiamin B) from Cassia siamea as the best potent Plasmepsin IV inhibitor. This compound has the potential candidate for future anti-malarial drugs. Cassiamin B had an affinity value of $-11.2 \mathrm{kcal} / \mathrm{mol}$ which was higher than PMIV's native ligand $(-3.8 \mathrm{kcal} / \mathrm{mol})$.

Keywords: Molecular docking, Anti-malarial, Cassia siamea, Cassiamin B, Plasmepsin IV
\end{abstract}

\section{Introduction}

In Indonesia, the prevalence of malaria tends to decrease from year to year. But there is still some province which was infected malaria often, especially in the east Indonesian region like Papua, Nusa Tenggara, and West Papua (the sum of positive malaria infections respectively 134,575; 17,388; and 7,450 people (Ministry of Health, 2019). Ironically, a plasmodial infection spread out and infected in the plant-rich places of Indonesia, including the endemic anti-malarial plant. Even though with its rich biodiversity, many people still do not know well about the function of the antimalarial plant and its mechanism. Traditional healers (tabib) had already been used specific plants to cure this plasmodial infection over generations but remains so many questions about its real efficacy and safety. At the present, science needs new alternatives to fight against this parasite. Those alternatives can be gathered, discovered, and validated by optimizing these specific plants, such as virtual screening for proven efficacy.

The Plasmodium parasite can be tricky. It is transferred freely into our body via several types of mosquito, such as the Anopheles genus. When this mosquito bites, which has a parasite on its body, the parasite rapidly flowthrough our body plasm. Our body consists of such a complex full package of hemoglobin in blood circulation. Hemoglobins play a major role in its intra-erythro-

\section{How to cite:}

Prakoso, A. H., Habiburrohman, M., Rohmatillah, W. N., Triatmoko, B., \& Nugraha, A. S. (2021). Molecular docking analysis of anti-malarial compounds as Plasmepsin IV inhibitor from targeted indonesian medicinal plants. The 3rd International Conference on Life Sciences and Biotechnology. NST Proceedings. pages 1-8. doi: 10.11594/

nstp.2021.0801 
cytic stages. The human malaria parasite, Plasmodium falciparum, degrades up to $80 \%$ of the hemoglobin in the infected red blood cell to provide nutrients for parasite growth and development. Parasite digestive vacuole, which is mediated by aspartic, cysteine, and metalloproteinases, plays a major role in its life cycle. Those enzymes are called Plasmepsins (PMs) family. Current evidence suggests that aspartic proteinases may be responsible for initiating this hemoglobin degradation pathway (Wyatt \& Berry, 2002). In P. falciparum, up to ten PMs have thus far been identified, namely PfPMs (Plasmodium falciparum Plasmepsins) 1, 2, 4-10 and PfHAP (Plasmodium falciparum Histo-Aspartic Proteinase) (Coombs et al.,2001; Liu, 2017). The blockade of these enzymes may result in parasite death. Plasmepsin I and II have already been identified and characterized. The present study also reported, a new aspartic proteinase, Plasmepsin IV also present within the digestive vacuole of the P. falciparum.

Table 1. Comparative properties of plasmepsins from the P.falciparum 3D7 strain in table form (Liu, 2017)

\begin{tabular}{lllllllllll}
\hline PM & Chr. & Pro & \multicolumn{3}{c}{ Zymogen } & \multicolumn{3}{c}{ Mature enzyme } \\
& & \#a.a. & \#a.a. & \%i.d. & MW (Da) & p1 & \#a.a. & \%i.d. & MW (Da) & p1 \\
\hline PM1 & 14 & 123 & 452 & 62 & 51,461 & 7,23 & 329 & 70 & 37,050 & 4,82 \\
PM2 & 14 & 124 & 453 & 61 & 51,481 & 5,29 & 329 & 69 & 36,915 & 4,62 \\
HAP & 14 & 123 & 451 & 52 & 51,694 & 8,23 & 328 & 59 & 36,979 & 4,97 \\
PM4 & 14 & 121 & 449 & - & 51,047 & 5,19 & 328 & - & 36,955 & 4,38 \\
PM5 & 13 & 83 & 590 & 25 & 68,481 & 7,66 & 440 & 25 & 50,844 & 6,50 \\
PM6 & 3 & 84 & 432 & 29 & 49,434 & 7,75 & 348 & 29 & 39,352 & 6,44 \\
PM7 & 10 & 76 & 450 & 28 & 52,329 & 8,44 & 374 & 28 & 43,315 & 6,09 \\
PM8 & 14 & 45 & 385 & 26 & 44,255 & 9,38 & 340 & 29 & 38,976 & 8,85 \\
PM9 & 14 & 212 & 627 & 27 & 74,184 & 9,63 & 402 & 25 & 46,970 & 9,28 \\
PM10 & 8 & 232 & 573 & 30 & 65,115 & 5,22 & 341 & 29 & 38,604 & 5,38 \\
\hline
\end{tabular}

PMs are distinct in structural and biochemical properties, such as molecular weight and isoelectric point as shown in Figure 1., also biological function (Figure. 2.). PMIV, PMI, PMII, and HAP cluster in the region of chromosome 14, and share a high amino acid sequence identity. These four PM paralogs were initially detected in the food vacuole (FV), an acidic organelle unique to the genus Plasmodium where degradation of hemoglobin of red blood cells (erythrocytes) occurs (Francis et al., 1994). PMs V until X share a low amino acid sequence identity with the FV PMs, and their sequence structures are distinct from each other and those of the FV PMs, indicating that there exist diverse biological functions and enzymatic features among the PM family members. Interestingly, the major parasitic role of the PM family appeared on PM IV as shown in Figure. 1. By knowing this information, this in-silico research aims to find novel compounds from listed plants as a potential anti-malarial drug. 


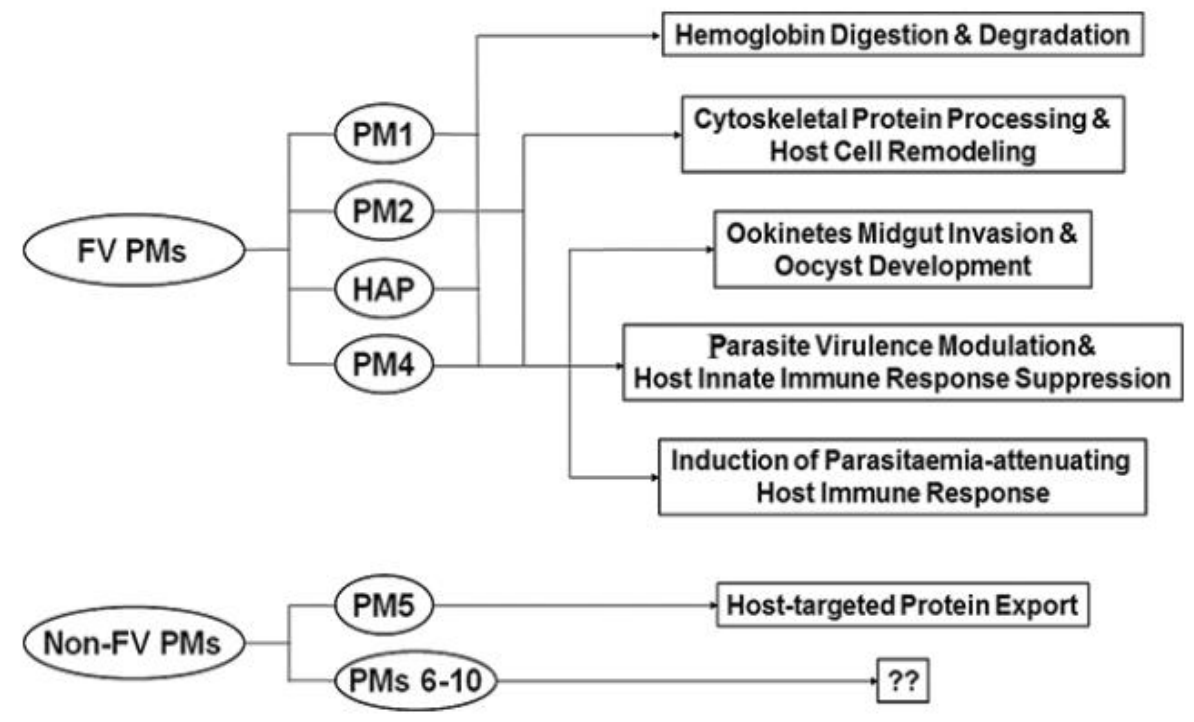

Figure 1. A diagram illustrating the connections of plasmepsins and their known biological functions (Liu, 2017; Banerjee et al., 2001)

\section{Material and Methods}

Preparation of Indonesian anti-malarial plants info

Approximately 238 compounds and their structure were gathered from many anti-malarial related journals, Indonesian medicinal plants book, Herb Index, which resumed carefully by DUDRG Anti-malarial Member.

\section{Target enzyme preparation}

Target enzyme (Plasmepsin IV) and its native ligand were in .pdb format and downloaded from RCSB PDB (www.rcsb.org). To find this enzyme, Protein Data Bank codenamed it as 5I70.

\section{Ligand preparation}

Plasmepsin IV native ligand was downloaded in format ".pdb" from packed enzyme set from Protein Data Bank online. About 238 compound structures were redrawn in ChemBioDraw Ultra 12.0 and minimized their energy using MM2 Minimize Energy with ChemBio3D Ultra 12.0. All compounds are saved in ".pdbqt" format to get docked. Saved .pdbqt formats codenamed as AM1 - AM238. AM means Anti-Malaria.

\section{Griding}

Searching the area of the docking process, ligand versus enzyme. Predicting that area was conducted by Autodock Tools, using the native ligand and the enzyme as a reference.

\section{Molecular Docking Protocol}

Virtual docking conducted using AutoDock Vina 1.1.2 software (Trott \& Olson, 2009), encrypted with a modified script from DUDRG virtual-screening leader. The redocking process showed affinity $=-3.8 \mathrm{kcal} / \mathrm{mol}$ with small RMSD $(1.435 \AA)$ in comparison to its crystal structure. The value of RMSD proves the validity of the docking protocols. The parameters used in the docking process are the free energy of binding $(\Delta G)$ as a marker of affinity. Amino acid's residue is also considered an interesting topic to discuss. 


\section{Results Visualization}

The docking results showed and visualized by PyMOL software. Visualization of docking result contains the interactions with its residual amino acid for additional further assay.

\section{Results and Discussion}

Table 1. List of best candidates and their affinity

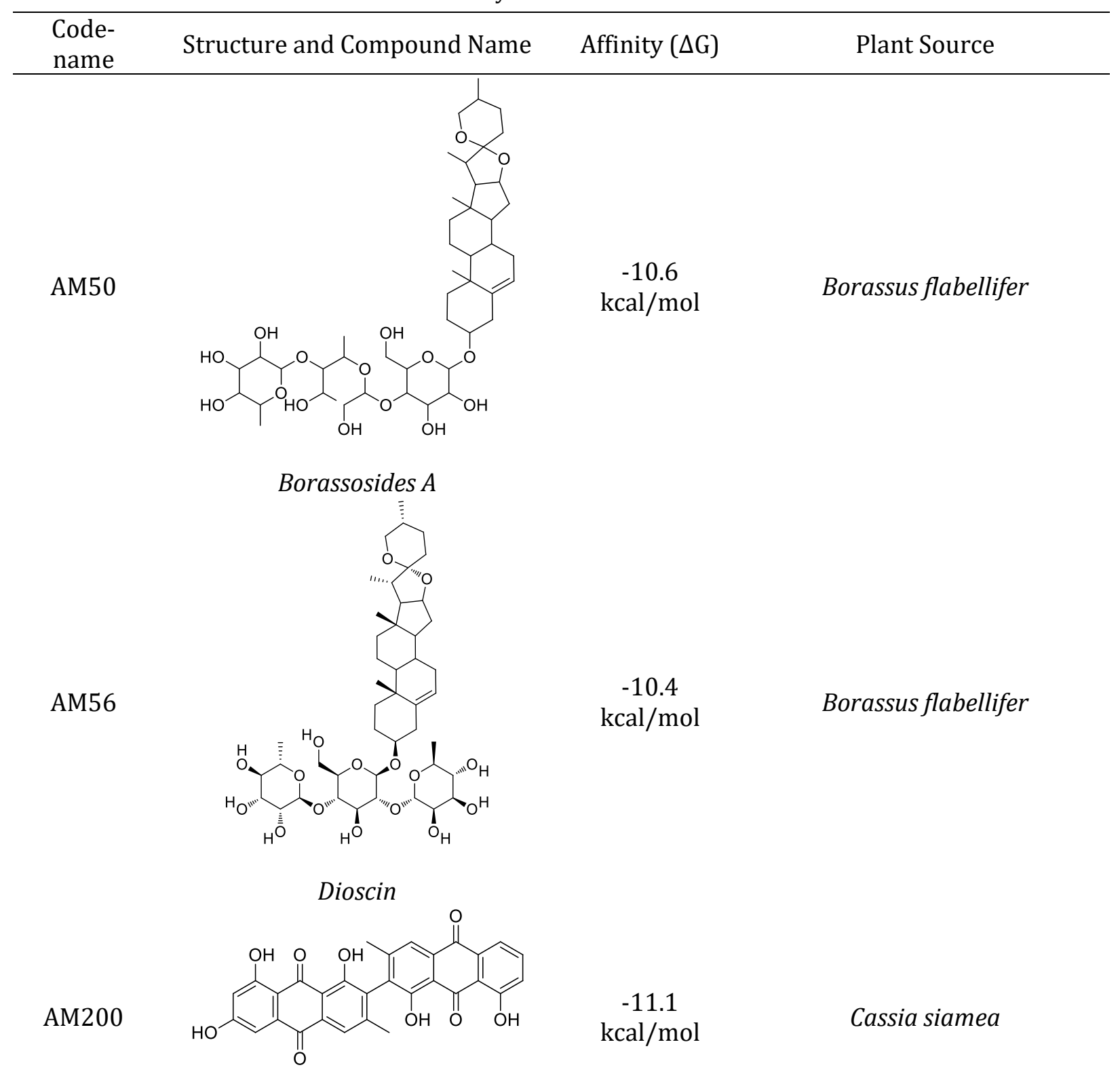

Cassiamin A 


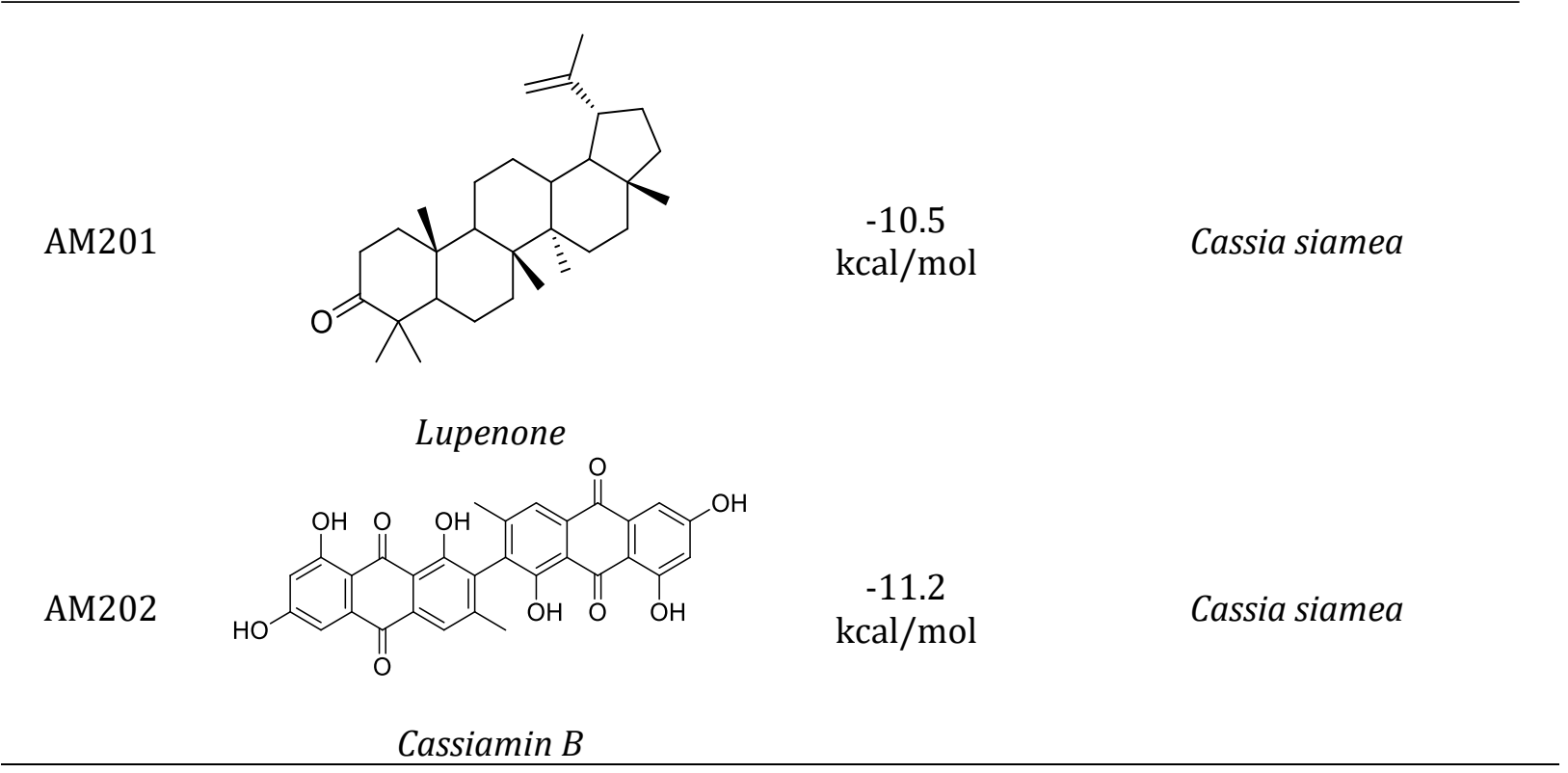

Table. 2 Amino acid residues

\begin{tabular}{ccccccc}
\hline Compund & Native & AM50 & AM56 & AM200 & AM201 & AM202 \\
\hline & & Gly-117 & & & & \\
& & Val-351 & Pro-113 & & & Phe-241 \\
$\begin{array}{c}\text { Amino acid's } \\
\text { Residue }\end{array}$ & Tyr-245 & Pro-291 & Tyr-245 & & Val-351 & Gly-78 \\
& & Lys-238 & & & \\
& & & & & \\
\hline
\end{tabular}

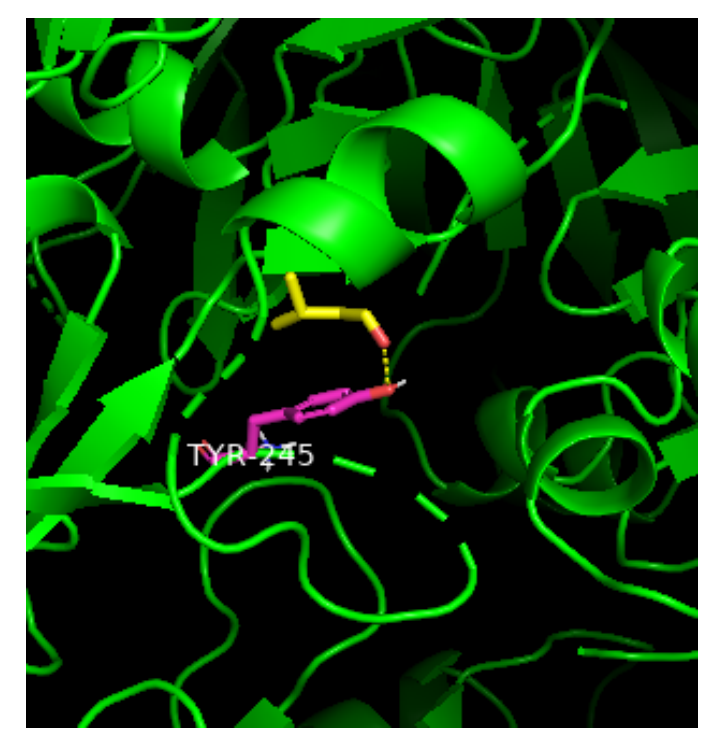

Figure 2. Hydrogen bonding of native ligand 
The docking process resulted in about 5 best candidates as anti-malarial compounds. They are AM50, AM56, AM200, AM201, and AM202 which have great potential affinity (Table 1.) with some amino acid residue (Table 2). The free energy binding $(\Delta G)$ of the five candidates is far-higher than the native ligand. It means the compounds are easy to dock with lesser energy to bind strongly with our receptor (protein target/PMIV). The more negative $\Delta \mathrm{G}$ shows a higher ligand affinity for the active site of the receptor (Du et al., 2016). This interaction happens due to residual amino acid binding and also some hydrogen bonding. The native ligand forms a hydrogen bond with Tyr245 residual amino acid (Figure. 3). To form this native ligand bonding, $-3.8 \mathrm{kcal} / \mathrm{mol}$ energy is needed. Acquired affinity showed that the energy needed for binding is too high and not so effective.

It seems the ligand-binding affinity is not determined by its amino acid residue similarity based on Table 2. There must be another factor (Pratama \& Aziz, 2019) like molecular weight, or some functional groups that play a role in anchoring the binding activities. The candidates show some great value of affinity. All of them, have an affinity ( $\Delta G$ score) lower than $-10 \mathrm{kcal} / \mathrm{mol}$, respectively three compounds from Cassia siamea (AM200, AM201, AM202), and the other two from Borassus flabellifer (AM50, and AM56). Each candidate showed different residual amino acid binding interactions.

Borassus flabellifer compounds consist of polycyclic hydrocarbon packed in high molecular weight. AM50 has some complex H-bonding interactions between its hydroxyl group to some residual amino acids (Gly-117, Val-351, Pro-291, and Lys-238). However, it needs $-10.6 \mathrm{kcal} / \mathrm{mol}$ to bind with the PMIV receptor. AM56, which has a different downside chain of AM50 also has some $\mathrm{H}$-bonding interactions between its hydroxyl groups with some residual amino acids (Pro-113, and Tyr-245) (As shown in Figure. 4 and Figure 5.). AM56 has stronger energy bonding than AM50 $(-10.4 \mathrm{kcal} / \mathrm{mol})$.

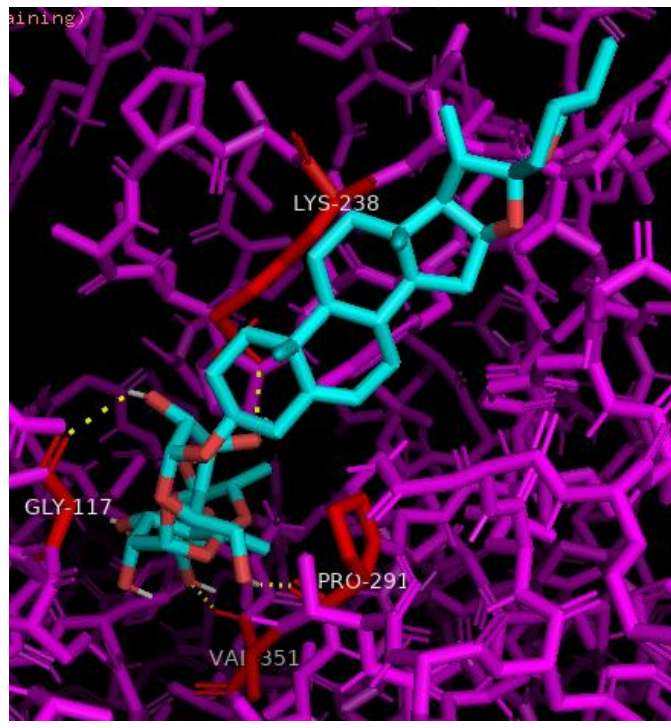

Figure 3. H-Bond Interaction of AM50 with its amino acids

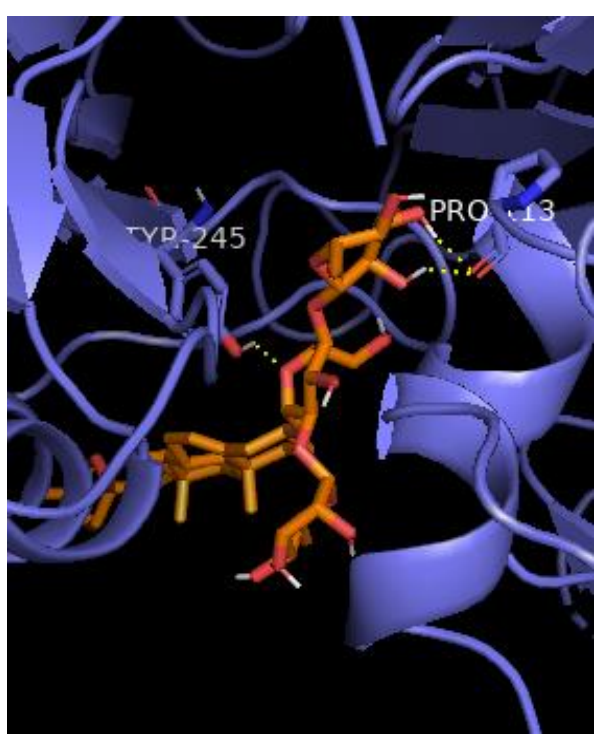

Figure 4. H-Bond Interaction of AM56 with its amino acids

Compounds from Cassia siamea seem to conflict with some interest. The affinity of three candidate compounds of this plant has far-lesser energy than the native Plasmepsin IV's ligand (in a range $-10.5 \sim-11.2 \mathrm{kcal} / \mathrm{mol}$ ). AM200, AM201, and AM202 have some similarities in the structure. They do have polycyclic groups with some electronegative atoms on the side chain. AM200 shows an H-bond from its carbonyl group with Asp-293. It also has an interesting affinity, -11.1 
$\mathrm{kcal} / \mathrm{mol}$. AM201 also shows an H-bond from its carbonyl group, but with a different amino acid residue, Val-351. Even though AM200 and AM201 have the same amount of H-bond, AM201 has a higher affinity than AM200 (-10.5 kcal/mol). AM202 has a strong binding activity due to the HBond of its carbonyl group supported by its hydroxyl in the left side of the tricyclic group, into a residual amino acid Phe-241. AM202 also has H-bond interaction (from another hydroxyl group) in the right tricyclic chain with Gly-78 as shown in Figure 6. This complexity makes ligand binds strongly inside the receptor (PMIV). Cassiamin B has a great potential affinity value (-11.2 $\mathrm{kcal} / \mathrm{mol}$ ). It only needs less energy to dock and bind to the Plasmepsin IV enzyme. AM202 shows great potential as a potent Plasmepsin IV inhibitor.

Although the uses of Cassiamin B (AM202) as anti-malarial were previously reported, its mechanism has not been studied. Therefore, this study can be used as an early explanation on the mechanism in which useful as QSAR study to develop these compounds as anti-malarial agents through Plasmepsin IV inhibitor. Another research (in vivo or in-vitro) could start to do some assay, and prove that AM202 is a potent anti-malarial drug.

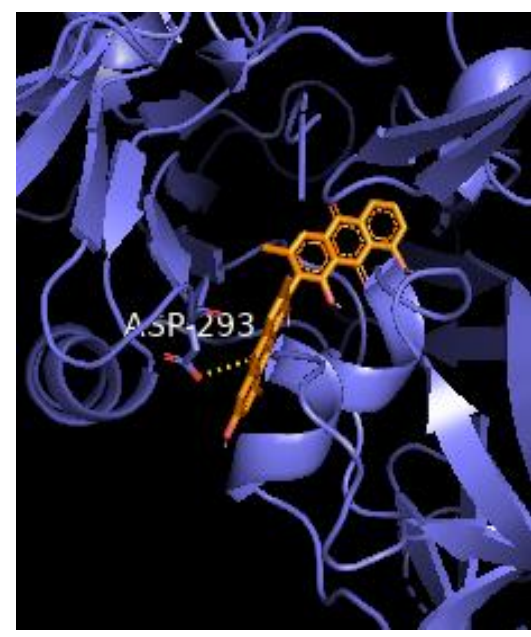

(a)

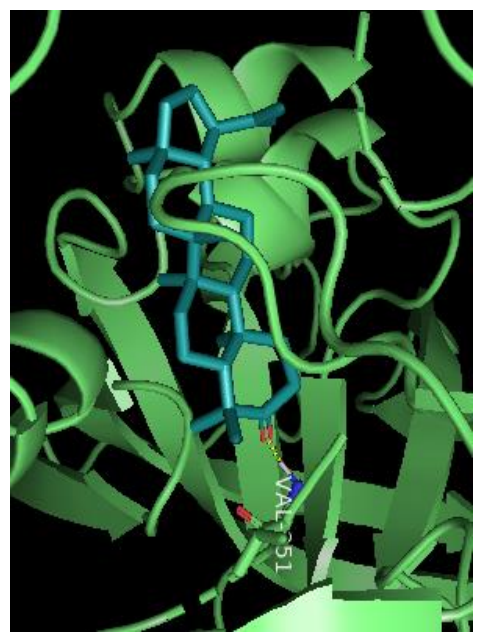

(b)

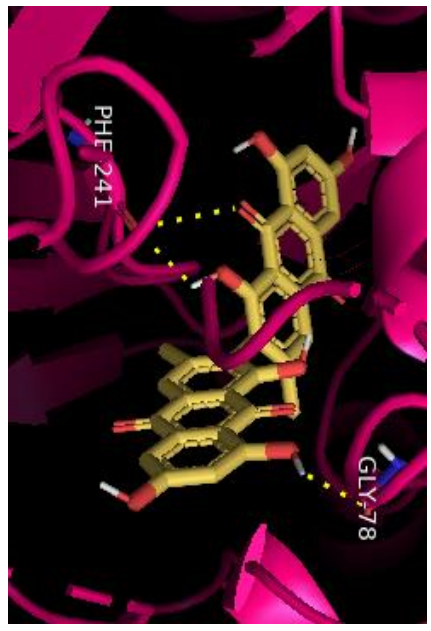

(c)

Figure 5. H-Bond Interaction beetwan some amino acids with (a) AM200; (b) AM201; (c) AM202

\section{Conclusion}

From the five best-resulted candidates, the potential value of affinity is shown on AM202, followed by AM200, AM50, AM201, and the least among candidates, AM56. The best candidate is indicated by its energy of bonding. The less energy it needed shows the better ligand becomes a potent anti-malarial drug.

Three compounds from Cassia siamea are the best candidates amongst 238 other compounds as a potential anti-malarial drug, respectively cassiamin A, lupenone, and cassiamin B. This insilico test provided initial basic information about affinity and should be proved by some bioguided assay.

\section{Acknowledgment}

Author thanks to University of Jember for the research support 


\section{References}

Banerjee, R., J. Liu, W. Beatty, L. Pelosof, M. Klemba, dan D. E. Goldberg. (2001). Four plasmepsins are active in the plasmodium falciparum food vacuole including a protease with an active-site histidine. https://doi.org/10.1073/pnas.022630099

Coombs, G. H., D. E. Goldberg, M. Klemba, C. Berry, J. Kay, J. C. Mottram, dan J. C. Mottram. (2001). Aspartic proteases of plasmodium falciparum and other parasitic protozoa as drug targets. Trends in Parasitology, 17(11), 532-537. doi: 10.1016/s14714922(01)02037-2

Du, X., Li, Y., Xia, Y-L., Ai, S-M., Liang, J., Sang, P., Ji, X-L., \& Liu, S-Q. (2016). Insights into protein - ligand interactions: mechanisms, models, and methods. International Journal of Molecular Science, 17(2), 1-34. doi: 10.3390/ijms17020144

Francis, S. E., Gluzman, Y., Oksman, A., Knickerbocker, A., Mueller, R., Bryant, M. L., Sherman, D. R., Russell, D. G., \& Goldberg, D. E. (1994). Molecular characterization and inhibition of a plasmodium falciparum aspartic hemoglobinase. The EMBO Journal, 1(2), 306317.

Liu, P. (2017). Plasmepsin: function, characterization and targeted antimalarial drug development. Open access peer-reviewed chapter, doi: $10.5772 / 66716$.

Ministry of Health. (2019). Data dan informasi profil kesehatan Indonesia 2018. Jakarta: Kementerian Kesehatan Republik Indonesia.

Pratama, M. R. F., \& Aziz, I. R. (2019). Molecular docking of bawang dayak (Eleutherine bulbosa) secondary metabolites as bacterial cell wall synthesis inhibitor. (June). 1st International Conference on Science and Technology, ICOST 2019, 2-3 May, Makassar, Indonesia.

Trott, O. dan A. J. Olson. (2009). Software news and update autodock vina: improving the speed and accuracy of docking with a new scoring function, efficient optimization, and multithreading

Wyatt, D. M., Berry, C. (2002). Activity and inhibition of plasmepsin iv, a new aspartic proteinase from the malaria parasite plasmodium falciparum. FEBS Letters, 513(2-3), 159-62. doi: 10.1016/s0014-5793(02)02241-x. 